\title{
Evaluation of novel assays for the detection of human papilloma virus in self-collected samples for cervical cancer screening
}

\author{
Q. Chen ${ }^{1}$, H. Du ${ }^{1,2}$, R. Zhang ${ }^{3}$, J.H. Zhao ${ }^{4}$, Q.C. Hu ${ }^{1,2}$, C. Wang ${ }^{1,2}$, \\ G.X. Wang ${ }^{1,2}$, J.L. Tang ${ }^{1,2}$ and R.F. Wu ${ }^{1,2}$ \\ ${ }^{1}$ Peking University Shenzhen Hospital, Shenzhen, China \\ ${ }^{2}$ Shenzhen Key Laboratory on Technology for Early Diagnosis of Major \\ Gynecological Diseases, Shenzhen, China \\ ${ }^{3}$ Jiangsu BioPerfectus Technologies, Jiangsu, China \\ ${ }^{4}$ Human Assisted Reproduction Center, Shanxi Women \& Children's Hospital, \\ China \\ Corresponding author: R.F. Wu \\ E-mail: wuruifang_1@163.com
}

Genet. Mol. Res. 15 (2): gmr.15027896

Received October 22, 2015

Accepted March, 18, 2016

Published June 24, 2016

DOI http://dx.doi.org/10.4238/gmr.15027896

\begin{abstract}
The aim of this study was to evaluate the performance of three new high-risk human papillomavirus (HPV) assays for primary cervical cancer screening, by using self-collected samples, and to identify an HPV assay that could overcome the major obstacles faced during large-scale population-based screening. Two hundred and ten women showing abnormal cervical cytology (and referred for a colposcopy) were recruited in this study. Self-collected samples obtained from all women were tested with the Cobas, Seq, and BioPerfectus Multiplex Real Time HPV assays; simultaneously, clinician-collected samples (from the same women) were tested with the gold-standard Cobas HPV assay. The results of all the assays were consistent. The sensitivity, positive predictive value, and negative predictive value for cervical intraepithelial neoplasia $2+(\mathrm{CIN} 2+)$ and $\mathrm{CIN} 3+$ were comparable
\end{abstract}


between the self-collected samples tested with the three new assays and the clinician-collected samples tested with the Cobas HPV assay $(\mathrm{P}>0.05)$. The single-genotype HPV load per sample did not differ significantly between the self- and clinician-collected samples ( $\mathrm{P}$ $=0.195)$. In conclusion, the results of this study demonstrated the applicability of the three new HPV assays for primary cervical cancer screening based on self-collection.

Key words: Cervical cancer; Human papillomavirus; Screening; Cervical intraepithelial neoplasia

\section{INTRODUCTION}

Cervical cancer is the third most common cancer in women, accounting for $13 \%$ of all cancers. Approximately 528,000 women were diagnosed with cervical cancer worldwide, resulting in 266,000 deaths, with $85 \%$ of the cases occurring in developed countries (Ferlay et al., 2015). Persistent high-risk human papillomavirus (HPV) infection is a prerequisite for the development of cervical cancer. Cervical cancer screening has successfully decreased its incidence and mortality (Fitzmaurice et al., 2013). However, several aspects of traditional cervical cancer screening programs are yet to be realized in low-resource countries.

China is the most populous country in the world; $70 \%$ of the Chinese population is currently living in rural regions. Cervical cancer incidence is typically highest in the rural areas of China (Yang et al., 2003). Many women living in low-resource regions or rural areas do not have access to regular screening for cervical cancer. Several population-based cervical cancer screening studies conducted over the past few decades have identified high-risk HPV DNA testing as an appropriate primary screen for cervical cancer in low-resource regions or countries (Qiao et al., 2008; Sankaranarayanan et al., 2009; Ronco et al., 2010). The results of 17 cross-sectional and population-based cervical cancer screening studies conducted in 30,371 women from various parts of China revealed that HPV DNA testing was a highly sensitive and moderately specific screening method for cervical intraepithelial neoplasia $3+(\mathrm{CIN} 3+)$ and higher types of cancer (Zhao et al., 2010).

The past 16 years have seen the introduction of vaginal self-collection for cervical cancer screening as a way to reach the medically under-served. The available literature suggests that self-sampling is more acceptable and has good performance, contributing to the increase in cervical cancer screening coverage (Arbyn et al., 2014). The clinical performance of self-sampling in the context of cervical cancer screening is currently being assessed using established assay platforms common to clinical laboratories. To date, very few studies have attempted to validate HPV testing using self-collected vaginal samples.

In this study, we have attempted to evaluate three new HPV assays for cervical cancer screening - the Cobas 4800 HPV Assay (Roche Molecular Systems, Risch-Rotkreuz, CA, USA), Seq HPV Assay (BGI Shenzhen, Shenzhen, China), and BMRT HPV Assay (BioPerfectus Technologies, Taizhou, China) - for self-collected samples. The cell count and viral load in the self- and clinician-collected samples were compared to prove the feasibility of self-sampling. 


\section{MATERIAL AND METHODS}

\section{Study population}

Two hundred and ten patients aged 18-56 years with abnormal cervical cytology ( $\geq$ atypical cells of undetermined significance [ASCUS]) and presenting a positive HR-HPV test (HC II assay) were recruited from the Peking University Shenzhen Hospital between September 2013 and December 2013. Signed informed consent forms were obtained from all participants after being informed about the goals and specific conduct of the study. The clinical trial was approved by the Institutional Review Board of the Peking University Shenzhen Hospital.

\section{Specimen collection}

Self-collected samples were obtained from each participant prior to their speculum examination using a cone-shaped brush (Qiagen, Venlo, Netherlands). After self-collection, a direct endocervical sample was obtained by a physician using a vaginal speculum, using the same type of brush. The clinician-collected endocervical sample was processed for the Cobas HPV assay. Self-collected samples were processed in the following order: the Cobas HPV assay (Roche Molecular Systems), Seq HPV Assay (BGI Shenzhen), BMRT HPV Assay (BioPerfectus Technologies).

\section{Cobas HPV assay}

Cobas HPV assay, an automated assay run on the Cobas 4800 system, is used to identify HPV DNA from among 14 high-risk HPV types. HPV DNA is isolated from a cell scraping and mixed with HPV DNA-specific primers and probes in reaction wells. The fluorescence produced by this reaction is then measured to determine the presence of HPV in the sample. The assay is designed to specifically identify HPV types 16 and 18 and concurrently detect the remaining 12 high-risk HPV types $(31,33,35,39,45,51,52,56,58,59,66$, and 68$)$. The assay was performed by laboratory staff trained by Roche Molecular Diagnostics by using the manufacturer protocols.

\section{SeqHPV assay}

SeqHPV assay is a HPV genotyping assay that uses multiplex PCR and next generation sequencing (BGI Shenzhen) to detect $14 \mathrm{HR}-\mathrm{HPV}$ types. The SeqHPV assay was used to amplify the approximately $150 \mathrm{bp}$ of the $H P V L 1$ gene using a series of unique primers. The human $\beta$-globin $(H B B)$ gene was used as the internal quality control (Yi et al., 2014).

\section{BMRT HPV PCR assay}

PCR primers and corresponding TaqMan probes were developed for the 21 most prevalent HPV types to amplify the HPV L1 gene, including 18 HR-HPV genotypes (HPV $16,18,26,31,33,35,39,45,51,52,53,56,58,59,66,68,73$, and 82), and 3 LR-HPV 
genotypes (HPV 6, 11, and 81). To control DNA quality and determine the relative viral copy numbers in the samples, a single-copy gene encoding DNA topoisomerase III (human TOP3) was amplified in the reaction.

\section{Statistical methods}

The association between categorical measures was assessed using the Pearson chisquare test. Kappa coefficients were used for analysis. The Wilcoxon signed-rank test was used to calculate the viral load and cell counts. All tests were performed at a significance level of 0.05 . The software platform SPSS v.17.0 (IBM, Armonk, NY, USA) was used for all analyses.

\section{RESULTS}

Of the 210 patients, at least one of the samples obtained from 13 patients were insufficient, and were excluded from the study. Samples from the remaining 197 patients (mean age, 39.3 years; range, $18-56$ years) were analyzed.

\section{Concordance of the four HPV assays}

We observed that results of the Cobas HPV assay of the clinician-collected endocervical samples (Cobas-clinician) were in agreement with the results of the Cobas HPV (Cobas-self), SeqHPV (Seq-self), and BMRT HPV (BMRT-self) assays of the selfcollected samples, with Kappa values of 0.78, 0.70, and 0.70, respectively. For the HPV16 or -18 positive samples, the concordance rates between the Cobas-clinician assay and the Cobas-self, Seq-self, and BMRT-self assays were 95.31\%, 92.18\%, and 96.88\%, respectively. The HR-HPV results of Cobas-self, Seq-self, and BMRT-self were consistent with those of Cobas-clinician by $95.40 \%$ (166/174), 94.83\% (165/174), and 95.40\% (166/174), respectively (Table 1$)$.

\footnotetext{
Table 1. Concordance between Cobas-Clinician and the three novel HPV assays.
}

\begin{tabular}{l|c|c|c|c|c}
\hline & \multicolumn{5}{|c}{ Cobas-Clinician } \\
\cline { 2 - 6 } & $\begin{array}{c}\text { HPV16 or } 18 \text { positive } \\
\text { concordance rate (\%) }\end{array}$ & $\begin{array}{c}\text { 14 HR-HPV positive } \\
\text { concordance rate }(\%)\end{array}$ & $\begin{array}{c}\text { Negative concordance } \\
\text { rate }(\%)\end{array}$ & Concordance rate (\%) & Kappa value \\
\hline Cobas-Self & $95.31(61 / 64)$ & $95.40(166 / 174)$ & $91.30(21 / 23)$ & $94.92(187 / 197)$ & 0.78 \\
\hline Seq-Self & $92.18(59 / 64)$ & $94.83(165 / 174)$ & $82.61(19 / 23)$ & $93.40(184 / 197)$ & 0.70 \\
\hline BMRT-Self & $96.88(62 / 64)$ & $95.40(166 / 174)$ & $78.26(18 / 23)$ & $93.40(184 / 197)$ & 0.70 \\
\hline
\end{tabular}

\section{Detection efficiency of four HPV assays for CIN2+ and CIN3+ cancers}

The sensitivity, positive predictive value (PPV), and negative predictive value (NPV) of the four HPV assays for screening cancers at CIN2+ and CIN3+ stages are summarized in Tables 2 and 3, respectively. The sensitivity, PPV, and NPV of the three new assays did not differ significantly from those of Cobas-clinician for CIN2+ and CIN $3+$ cancers. 
Table 2. Sensitivity, positive predictive value (PPV), and negative predictive value (NPV) of four human papilloma virus (HPV) assays for cervical intraepithelial neoplasia $2+(\mathrm{CIN} 2+)$.

\begin{tabular}{l|c|c|c|c|c|c}
\hline & Sensitivity (\%) & P value & PPV (\%) & P value & NPV (\%) & P value \\
\hline Cobas-Clinician & $95.24(80 / 84)$ & - & $45.98(80 / 174)$ & - & $82.61(19 / 23)$ & - \\
\hline Cobas-Self & $92.86(78 / 84)$ & 0.75 & $46.43(78 / 168)$ & 0.93 & $79.31(23 / 29)$ & 0.76 \\
\hline Seq-Self & $94.04(79 / 84)$ & 0.73 & $46.63(76 / 163)$ & 0.91 & $85.71(24 / 28)$ & 0.76 \\
\hline BMRT-Self & $95.24(80 / 84)$ & 1.00 & $45.56(82 / 180)$ & 0.94 & $88.24(15 / 17)$ & 0.62 \\
\hline
\end{tabular}

Table 3. Sensitivity, positive predictive value (PPV), and negative predictive value (NPV) of four human papilloma virus (HPV) assays for cervical intraepithelial neoplasia $3+(\mathrm{CIN} 3+)$.

\begin{tabular}{l|c|c|c|c|c|c|c}
\hline & Sensitivity (\%) & P value & PPV (\%) & P value & NPV (\%) & P value \\
\hline Cobas-Clinician & $98.00(49 / 50)$ & - & $28.16(49 / 174)$ & - & $95.65(22 / 23)$ & - \\
\hline Cobas-Self & $96.00(48 / 50)$ & 0.56 & $28.57(48 / 168)$ & 0.93 & $93.10(27 / 29)$ & 0.69 \\
\hline Seq-Self & $96.00(48 / 50)$ & 0.56 & $28.40(48 / 169)$ & 0.96 & $92.85(26 / 28)$ & 0.67 \\
\hline BMRT-Self & $96.00(48 / 50)$ & 0.56 & $27.22(49 / 180)$ & 0.84 & $94.12(16 / 17)$ & 0.83 \\
\hline
\end{tabular}

\section{Comparison of the HPV load and cell count in self- and clinician-collected samples}

We compared the single-genotype HPV load and cell counts between self-collected and clinician-collected samples using BMRT HPV assays; we observed no significant changes in the single-genotype HPV load per sample between the self- and clinician-collected samples $(\mathrm{P}=0.195)$. However, self-collected samples had significantly higher cell counts than cliniciancollected samples $(\mathrm{P}<0.001)$ (Table 4; Wilcoxon signed-rank test).

Table 4. Wilcoxon signed-rank test.

\begin{tabular}{l|c|c}
\hline BMRT assay & Single genotype HPV load (copies/sample) $(\mathrm{N}=83)$ & Cell count $(\mathrm{N}=83)$ \\
\hline Self-Median & $47,545.2$ & $22,680.3$ \\
\hline Clinician-Median & $32,183.9$ & $2,156.3$ \\
\hline P value & 0.195 & $<0.001$ \\
\hline
\end{tabular}

\section{DISCUSSION}

In this study, we focused on the clinical application of three self-sampling-based HPV assays for cervical cancer screening, in order to identify an HPV assay that could overcome the major obstacles faced by self-sampling, such as throughput, cost, sensitivity, and compatibility, in order to improve their usability in large-scale screening programs.

The Cobas HPV assay was approved by the FDA in April 2011 for screening patients aged 21 years and above with abnormal cervical cytology and for adjunct use in women aged 30 years and above with normal cervical cytology, to assess the presence or absence of highrisk HPV genotypes. The Cobas HPV test and Cobas 4800 clinical system were validated in the ATHENA trial (Wright et al., 2015). The Cobas HPV test was approved by the FDA in 2014, as the only HPV assay providing specific genotyping information for the high-risk HPV types 16 and 18, while simultaneously reporting the 12 other high-risk HPV types as a pooled result in a single run from one patient sample. However, very few studies have reported the use of the Cobas HPV assay in cervical cancer screening by using self-collected samples.

A high-throughput HPV genotyping assay based on multiplex PCR and nextgeneration sequencing (NGS) technology was developed by BGI, China in 2014. This assay 
could be used for large-scale screening as the maximum throughput of this assay is $>1,200$ samples/run. Moreover, this assay was optimized to control the cost at $<\$ 5.00 /$ case. This assay could be used to detect the presence or absence of one or more 14 HR-HPV types, using the human $\beta$-globin gene $(H B B)$ as the internal quality control (Yi et al., 2014).

The BMRT HPV assay was developed by BioPerfectus Technologies in 2014 to detect 18 HR-HPV genotypes and three LR-HPV genotypes. This test was approved by the Chinese FDA in 2015. This test used a single-copy gene encoding DNA topoisomerase III (human TOP3) as the internal quality control, as well as to determine the relative viral copy numbers in the samples.

Previous studies have demonstrated that the sensitivity of a self-collected sample was comparable to that of a physician-obtained specimen when polymerase chain reaction (PCR)based technology was used for the analysis (Belinson et al., 2010; Du et al., 2011; Belinson et al., 2012). In this study, clinician-collected specimens analyzed using the Cobas HPV assay comprised the control group; these samples were used to validate and confirm the comparable performance of the three new assays using self-collected samples to those of assays conducted on clinician-obtained specimens. First, we observed a very strong agreement between the results of three novel HPV assays for a self-collected sample and that of a Cobas HPV assay for a gold-standard physician-collected endocervical sample. Furthermore, the kappa, positive concordance, and negative concordance values for analysis of the self-collected samples with the three HPV assays were comparable to those for analysis of the clinician-collected samples with the Cobas HPV assay. Second, the sensitivity, PPV, and NPV of the novel assay-tested self-collected samples were similar to those of the Cobas HPV assay-tested clinician-collected samples for CIN2+ and CIN3+ $(\mathrm{P}>0.05)$.

Previous studies have postulated that self-collected samples contain insufficient vaginal and cervical cells and HPV load, resulting in detection failure. We validated this hypothesis using the BMRT HPV assay. We found that the cell counts were significantly higher in self-collected samples than in clinician-collected samples. However, we attributed this to a greater number of vaginal cells, and not cervical cells, being collected by the patients. However, the HPV load was comparable between the self- and clinician-collected samples. Therefore, we concluded that vaginal self-sampling could be feasible for HR-HPV testing using a PCR-based assay.

The most significant strength of this study was that the three HR-HPV assays were performed in self- and clinician-collected samples obtained from over 210 women. However, this study also has some limitations: first, a population-based design was not used for cervical cancer screening, and second, very few of the samples were deemed insufficient for analysis.

In conclusion, this study was used to demonstrate the performance of three new assays in detecting HPV infection in self-collected samples. Moreover, as the three HPV assays can report type-specific HPV, they allow for the selection of high-risk types as a secondary screen to further improve the specificity of the management algorithm. The self-collection of samples and the three HPV novel assays could help ensure the centralized, high-throughput, sensitive, low-cost-per-case processing of samples obtained from individuals at high risk of cervical cancer within a short period of time.

\section{Conflicts of interest}

The authors declare no conflict of interest. 


\title{
ACKNOWLEDGMENTS
}

\author{
Research supported by the Shenzhen Municipal Science and Technical Innovation \\ Committee (\#JCYJ20120619145419556 and \#GJHZ 20130417100103783).
}

\section{REFERENCES}

Arbyn M, Verdoodt F, Snijders PJ, Verhoef VM, et al. (2014). Accuracy of human papillomavirus testing on self-collected versus clinician-collected samples: a meta-analysis. Lancet Oncol. 15: 172-183. http://dx.doi.org/10.1016/S1470$\underline{2045(13) 70570-9}$

Belinson JL, Hu S, Niyazi M, Pretorius RG, et al. (2010). Prevalence of type-specific human papillomavirus in endocervical, upper and lower vaginal, perineal and vaginal self-collected specimens: Implications for vaginal self-collection. Int. J. Cancer 127: 1151-1157. http://dx.doi.org/10.1002/ijc.25144

Belinson JL, Du H, Yang B, Wu R, et al. (2012). Improved sensitivity of vaginal self-collection and high-risk human papillomavirus testing. Int. J. Cancer 130: 1855-1860. http://dx.doi.org/10.1002/ijc.26202

Du H, Yi J, Wu R, Belinson SE, et al. (2011). A new PCR-based mass spectrometry system for high-risk HPV, part II: clinical trial. Am. J. Clin. Pathol. 136: 920-923. http://dx.doi.org/10.1309/AJCPJDAORUY4EYR6

Ferlay J, Soerjomataram I, Dikshit R, Eser S, et al. (2015). Cancer incidence and mortality worldwide: sources, methods and major patterns in GLOBOCAN 2012. Int. J. Cancer 136: E359-E386.

Fitzmaurice C, Dicker D, Pain A, Hamavid H, et al.; Global Burden of Disease Cancer Collaboration (2015). The global burden of cancer 2013. JAMA Oncol. 1: 505-527. http://dx.doi.org/10.1001/jamaoncol.2015.0735

Qiao YL, Sellors JW, Eder PS, Bao YP, et al. (2008). A new HPV-DNA test for cervical-cancer screening in developing regions: a cross-sectional study of clinical accuracy in rural China. Lancet Oncol. 9: 929-936. http://dx.doi. org $/ 10.1016 / \mathrm{S} 1470-2045(08) 70210-9$

Ronco G, Giorgi-Rossi P, Carozzi F, Confortini M, et al.; New Technologies for Cervical Cancer screening (NTCC) Working Group (2010). Efficacy of human papillomavirus testing for the detection of invasive cervical cancers and cervical intraepithelial neoplasia: a randomised controlled trial. Lancet Oncol. 11: 249-257. http://dx.doi. org/10.1016/S1470-2045(09)70360-2

Sankaranarayanan R, Nene BM, Shastri SS, Jayant K, et al. (2009). HPV screening for cervical cancer in rural India. $N$. Engl. J. Med. 360: 1385-1394. http://dx.doi.org/10.1056/NEJMoa0808516

Wright TC, Stoler MH, Behrens CM, Sharma A, et al. (2015). Primary cervical cancer screening with human papillomavirus: end of study results from the ATHENA study using HPV as the first-line screening test. Gynecol. Oncol. 136: 189197. http://dx.doi.org/10.1016/j.ygyno.2014.11.076

Yang L, Huangpu XM, Zhang SW, Lu FZ, et al. (2003). [Changes of mortality rate for cervical cancer during 1970's and 1990's periods in China]. Zhongguo Yi Xue Ke Xue Yuan Xue Bao 25: 386-390.

Yi X, Zou J, Xu J, Liu T, et al. (2014). Development and validation of a new HPV genotyping assay based on nextgeneration sequencing. Am. J. Clin. Pathol. 141: 796-804. http://dx.doi.org/10.1309/AJCP9P2KJSXEKCJB

Zhao FH, Lin MJ, Chen F, Hu SY, et al.; Cervical Cancer Screening Group in China (2010). Performance of high-risk human papillomavirus DNA testing as a primary screen for cervical cancer: a pooled analysis of individual patient data from 17 population-based studies from China. Lancet Oncol. 11: 1160-1171. http://dx.doi.org/10.1016/S1470$\underline{2045(10) 70256-4}$ 